\section{Novel Developments in High-Frequency Micro-Ultrasound Imaging}

\author{
Tom Little \\ VisualSonics Inc., Toronto, Ontario, Canada \\ tlittle@visualsonics.com
}

With the mapping of the mouse genome, the growing sophistication in transgenic sciences creating complex mouse models of disease, and the demand to study disease in vivo, there has been a corresponding increase in the demand for and development of preclinical imaging modalities. Clinical ultrasound operating in the $2-12 \mathrm{MHz}$ range is a well established clinical imaging modality, accounting for more than one-third of all imaging procedures performed in North America. The simplicity, ease of use, speed, and safety of ultrasound have led to its significant role in diagnosis, treatment assessment, follow-up, and guidance of therapy in clinical applications. Ultrasound imaging is used routinely in its B-Mode imaging mode to report on soft tissue structures. It's also used in its Doppler modes for the measurement of blood velocity in fast-flowing targets such as the cardiovascular system, in slow-flowing applications such as quantifying blood flow and in vascular architectures within tumors. While powerful and flexible as a clinical modality, ultrasound's low spatial resolution is not sufficient to be useful as a modality to assess the small structures and biological targets of the mouse model, especially when considering early stage disease development or embryonic studies of small animal models.

\section{"Resolution Revolution"}

The development of high-frequency micro-ultrasound (20 $\mathrm{MHz}+$ ) - or ultrasound biomicroscopy (UBM) - applies all the benefits of ultrasound as a modality to the broad range of applications required in preclinical research. High-resolution micro-ultrasound operates in the frequencies of $35-80 \mathrm{MHz}$ with spatial resolution down to 30 microns and real-time temporal resolution. The novel nature of the technology lies in the fact that these very high resolutions (i.e., visualizing anatomical structures and micro blood flow) were achievable, but only with low penetration $(2-3 \mathrm{~cm})$. While limited for clinical applications, this depth of penetration is very appropriate for small animal research models-making this imaging platform ideally suited for visualizing the small anatomical

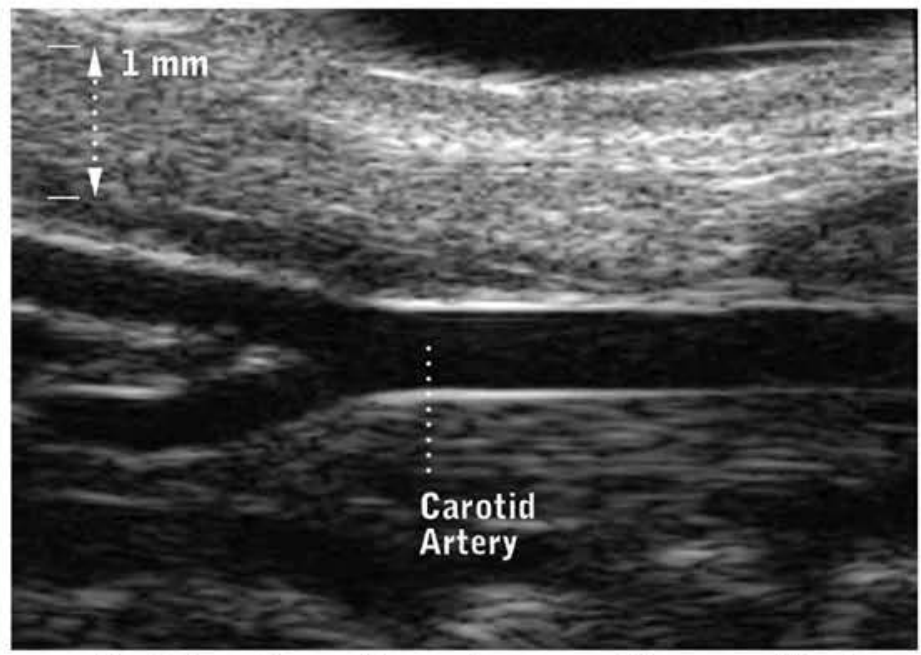

Fig. 1 Micro-ultrasound image of mouse carotid artery, enabling the viewing and measurement of plaque development and vessel wall thickness in real-time. structures and blood flow (see Figure 1) that preclinical researchers need to evaluate phenotype and disease progression in vivo.

Micro-ultrasound is used routinely for assessment of soft tissue targets in abdominal imaging, cardiovascular imaging, confirmation of pregnancy and soft tissue tumor studies in mouse models. Since the technology is non-invasive and without bioeffects, the same animal can be imaged frequently in longitudinal studies that monitor the development of disease expression. Therapeutic efficacy can also be studied using this modality so as to monitor the progression or regression of disease.

\section{Cancer Applications}

The mouse is a popular biological model for cancer research. The need to assess early stage tumor development, growth and metastases, burden and flow and micro-vasculature parameters are important elements that lead to better understating of the nature of disease as well as therapeutic efficacy. Micro-ultrasound allows for real-time visualization of soft tissue tumors from early stage developments ( 100 microns). Tumors can be quantified in 2 - and 3-dimensions quickly and non-invasively (see Figure 2). Using Power Doppler mode, micro-vasculature details can be assessed and also visualized and measured in $3 \mathrm{D}$. Tumor and tumor vascular growth and architecture measures can be created and monitored longitudinally.

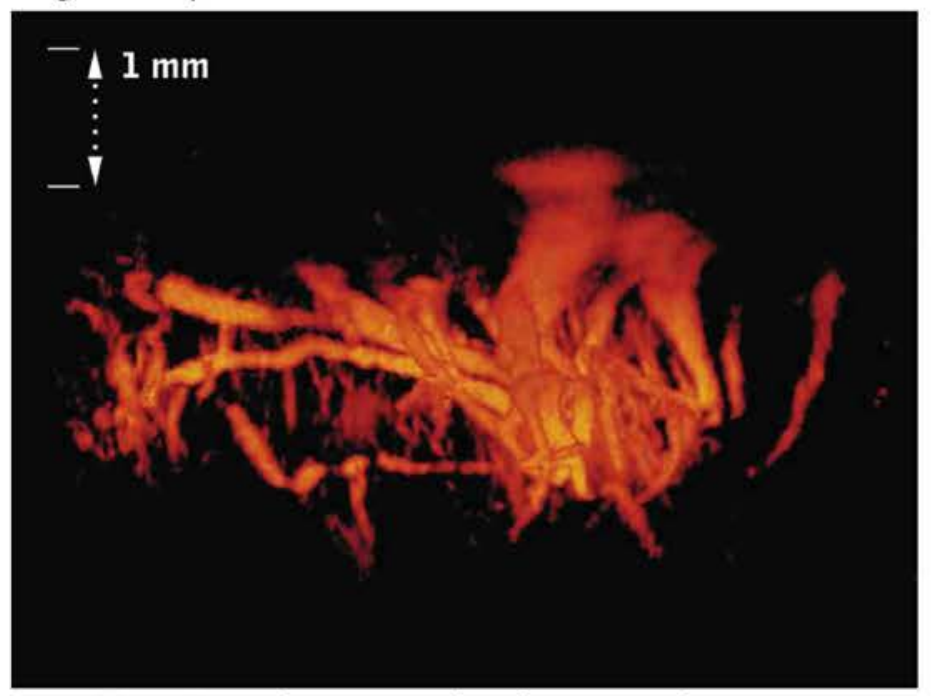

Fig. $23 D$ vascular structure of a melanoma growth in a mouse on its third day of growth.

With the development of targeted contrast agents, molecular imaging functionality is added to this platform as intra-vascular contrast agents can be targeted at key expression targets for disease areas of interest and then measured in vivo. Much work has begun to apply micro-ultrasound to anti-angiogeneic studies, especially with the development of such therapeutic agents in preclinical models.

\section{Cardiovascular Applications}

As the heart of the adult mouse beats at $450-600$ beats per minute and is a few millimeters in size, the VisualSonics' Vevo 770 has been developed to provide the spatial and temporal resolution required to visualize and quantify these cardiovascular phenotypes. In addition to B-Mode imaging, which provides frame rates of up to $240 \mathrm{~Hz}$ and spatial resolution to 30 microns, a line-based technique called EKV (ECG-based kilohertz reconstruction) allows researchers to visualize and measure the detailed beating adult mouse heart - at up to 1,000 frames/second. 


\section{Custom Microscopy Supplies} From

M. E. Taylor Engineering, Inc.

- SEM Light Pipe Scintillators, styles for Leo \&Cambridge SEMS and custom products

- Scintillators: ITO Gold, Phosphor, YAG and YAP from $\$ 89$

- Recoating of Light Pipes from $\$ 150$

- Conductive Adhesive Products Silver paint, pens and epoxy Carbon paint, tape and tabs

- ProScope and Dino Microscopes and accessories

\section{Phone (301) 774-6246}

See our products on the Web @ www.semsupplies.com

\section{Why have 1,000 scientists in 25 countries selected Minus $\mathrm{K}$ vibration isolators?}

Because Minus K vibration isolation systems deliver $10 x$ to $100 x$ better performance than high-performance air systems, without air, and for air table prices.

\section{Visit www. minusk.com for more information...}

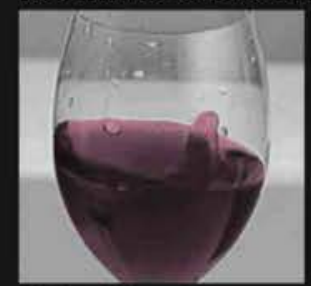

Without Minus K

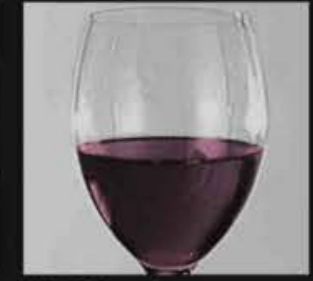

With Minus K

VIBRATION ISOLATION BY:

\section{minus $\mathbf{k}^{\circ}$ TECHNOLOGY}

420 S. Hindry Ave., Unit E • Inglewood, CA 90301 USA Phone: $310-348-9656$ - Fax: $310-348-9638$

sales@minusk.com•www.minusk.com

\section{I can see clearly now the contamination is gone.}

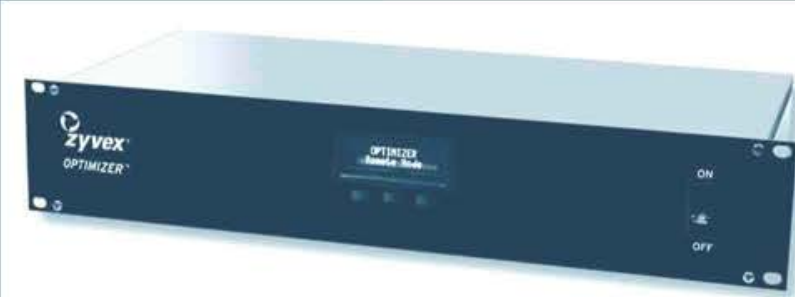

C( Patents Pending

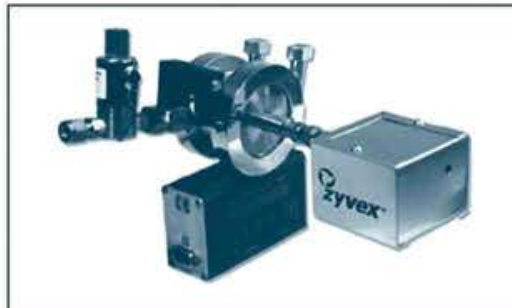

If you're fighting carbon deposition, you know that success is determined by the difference between clean - and really clean.

For better images, faster cleanup, reliability, and ease-of-use, turn to the Zyvex@ Optimizer ${ }^{\mathrm{TM}}$ for the ultimate solution for Scanning Electron Microscopes, Focused Ion Beam Systems, and other vacuum systems. You can use the Optimizer ${ }^{T M}$ for materials science, metrology, nano probing, micro and nanoscale research, and integration into micro robotics and micro manipulation systems.

Stop looking at contamination and start looking at the real thing. Turn to the solutions experts. We're providing nanotechnology solutions - today. ${ }^{\top M}$

For more information, please call 877-998-3999 Ext. 271 or visit our website at www.zyvex.com

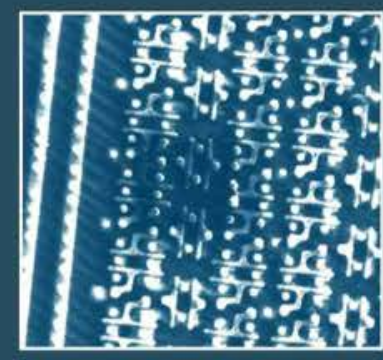

The black square shown on this microchip is polymerized hydrocarbon buildup. This is a result of continuous scanning at $60 \mathrm{kX}$ magnification for one hour.

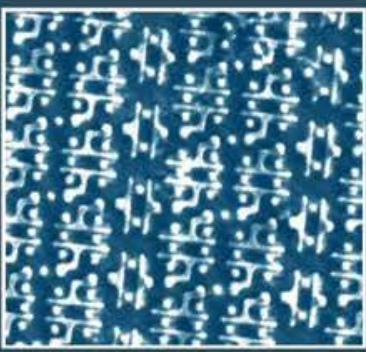

$2 \mu \mathrm{m}$

Using the Zyvex@ Optimizer ${ }^{T M}$ for cleanup, and shifting to a clean site, the same microchip was exposed to one hour of continuous scanning. No detectable amount of hydrocarbon buildup is shown. 
Due to its high-resolution, micro-ultrasound can be applied to new areas of cardiovascular research previously not investigated due to the limitations of the available imaging tools including:

- Cardiac ischemic mice models to determine wall motion and visualization of all segments of myocardium;

- Embryonic lethal mouse models in utero to visualize the atrial and ventricular function from embryonic day 6.5 onward and perform Doppler of outflow tracts;

- Cardiac models affecting right-sided cardiac anatomy including tricuspid and pulmonary valve anatomy and function;

- Cardiac models affecting diastolic function using mitral valve Doppler;

- Guidance of injection procedures including image-guided injections into the mouse and rat myocardium for cardiovascular stem cell research, introduction of therapeutics, and LV-guided injection for perfusion;

- In Vivo Monitoring and quantifying of therapeutic efficacy longitudinally, from early stages of mouse development; and

- Monitoring of surgical implants including guidance of and monitoring of effective placement and function of shunts, etc. non-invasively and longitudinally.

\section{Complete Pre-Clinical Solution}

With a spatial resolution of 30 microns and the small size of the instrument's scanheads, detailed imaging and phenotyping of many small animal models is possible - including embryonic mice, zebra fish, and chick embryos, all important models for cardiovascular and developmental modeling of human disease. An integrated small animal rail system and handling apparatus allows researchers to perform detailed assessments and imaging procedures with accuracy and reproducibility. The system also allows for the physiological monitoring of the animal including capture of temperature, ECG, an integrated compact anesthesia system, and controlled warming of the animal during imaging.

The approach to data analysis is also research-centric providing open and easy access to raw image and measurement data and allowing for a variety of analytic and measurement tools for quantifying phenotypes.

\section{Novel applications for Image-Guided Injections and Inter- ventions}

As in clinical applications, micro-ultrasound, because it is a real-time modality, has been adopted for guiding injections for small animal procedures in neurobiology, stem cell therapy, and gene therapy applications. Neurobiologists use the technology to guide targeted injections in utero into cranial cavities in mouse models. DNA and genetic materials can be introduced or extracted in utero and then the phenotypes can be monitored through development, birth, and neonatal growth. Developmental studies have successfully used the Vevo to guide injections into targets in the developing mouse embryo in utero. Recent developments have also included using the Vevo to guide closed-chest injections into the myocardium for cardiac stem cell regeneration therapy. This application has replaced a previous technique involving complex cardiovascular surgery and imprecise injections.

\section{The Next Application: Contrast-Enhanced and Molecular Imaging at High Frequencies}

Ultrasound contrast agents or micro bubbles can compliment and extend the utility of micro-ultrasound by enhancing imaging modes and allowing for molecular imaging of disease biomarkers.

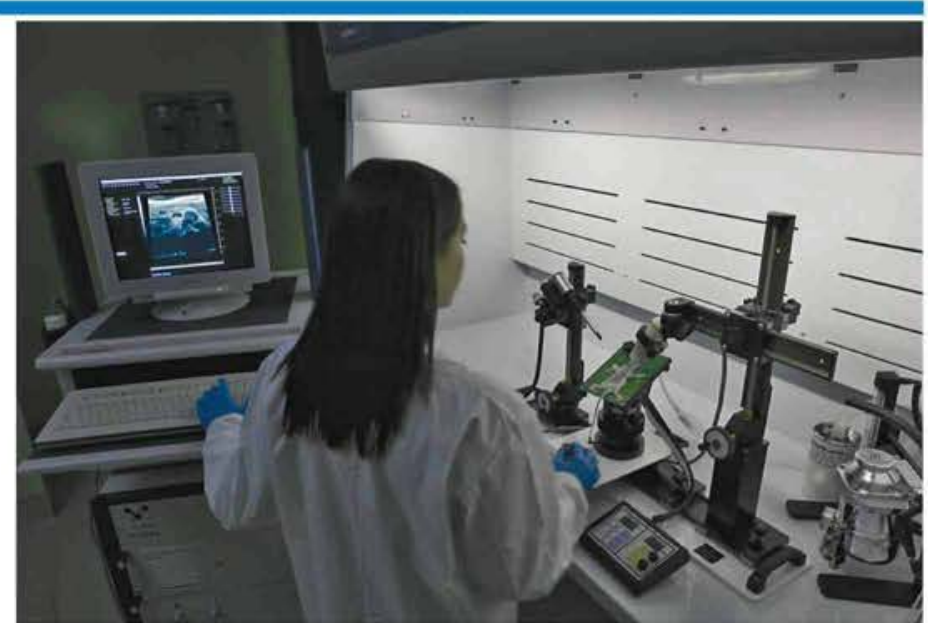

The VisualSonics' Vevo 770 "system.

Contrast agents are introduced into the blood pool of the mouse through intravenous injection and can be used for a number of key applications. As an image enhancing agent, micro bubbles can enhance the micro-ultrasound signal so as to visualize difficult to distinguish targets such as damaged myocardial borders for perfusion studies. As a targeted agent, a micro bubble can have specific ligands bound to the surface and introduced into the blood stream so that they bind only to that unique target site. Such is the case with VEGFR2, a popular biomarker for angiogenesis, or P-Selectin, another biomarker for the expression of inflammation. As such, the presence of these biomarkers can be detected in straightforward, reproducible procedures and the presence of the targeted agent can be quantified to provide relative quantification of the biomarker expression.

\section{Micro-Ultrasound: The Fastest Growing Preclinical In Vivo Imaging Modality}

In just over three years, VisualSonics has sold and activated over 200 micro-ultrasound systems for use globally in leading academic and pharmaceutical companies. This enabling technology - the Vevo 770 high-resolution micro-ultrasound system - is facilitating novel research in cardiac and vascular disease, cancer, stem cell research and developmental biology. When compared to other imaging options, only micro-ultrasound has the combination of high-resolution, real-time imaging, in vivo detection and analysis, reasonable cost, ease-of-use, portability and no negative biological effects. With the addition of contrast-enhanced and targeted contrast imaging, micro-ultrasound now provides a single preclinical micro-imaging platform for real-time anatomical, functional (i.e. blood flow) and now molecular imaging and analysis.

\section{Select References:}

Noninvasive Localization of Nuclear Factor of Activated T Cells c1-/- Mouse Embryos by Ultrasound Biomicroscopy-Doppler Allows Genotype-phenotype Correlation. Phoon CK et al., J Am Soc Echocardiogr. 2005 Dec;18(12):1415-21

Image-Guided Cardiac Cell Delivery Using High-Resolution Small-Animal Ultrasound. Gambhir S. et al, Molecular Therapy; 2005 Aug 16;

A new three-dimensional ultrasound microimaging technology for preclinical studies using a transgenic prostate cancer mouse model. Lacefield JC. Et al, Cancer Res. 2005 Jul 15;65(14):6337-45.

Noninvasive measurement of abdominal aortic aneurysms in intact mice by a high-frequency ultrasound imaging system. Wang YX et al., Ultrasound Med Biol. 2005 Jun; 31(6):745-9.

Abnormal cardiac inflow patterns during postnatal development in a mouse model of Holt-Oram syndrome.gFoster FS., et al., Am J Physiol Heart Circ Physiol, 2005 Apr 22

Ultrasonic detection and developmental changes in calcification of the placenta during normal pregnancy in mice, Adamson SL. et al, Placenta, 2005 Feb-Mar; 26(2-3):129-37. 

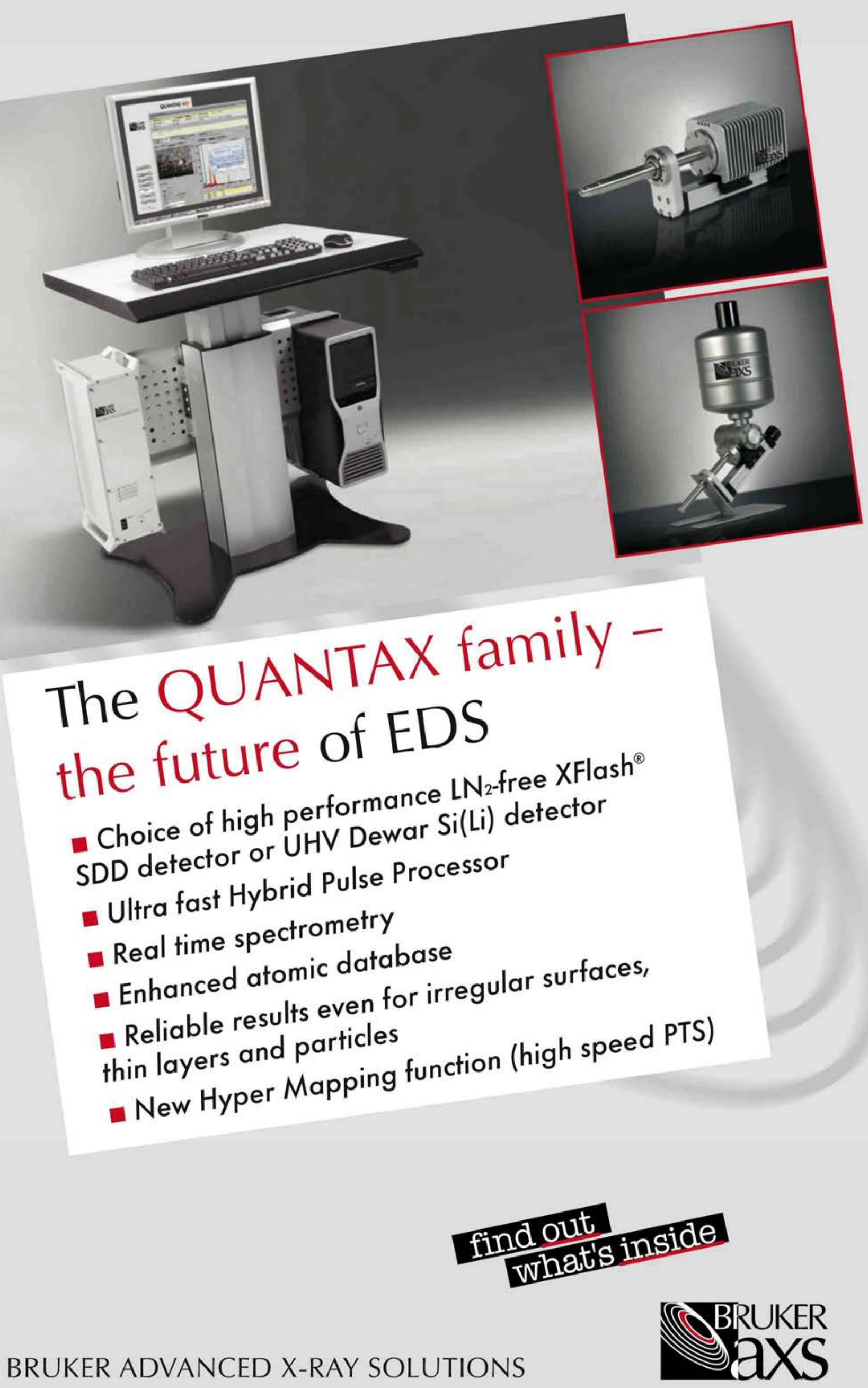